\title{
Subgingival debridement is effective in treating chronic periodontitis
}

\author{
Is subgingival debridement clinically effective in people who have chronic \\ periodontitis?
}

\begin{abstract}
Van der Weijden GA, Timmerman MF. A systematic review on the clinical efficacy of subgingival debridement in the treatment of chronic periodontitis. J Clin Periodontol 2002; 29(suppl. 3): S55-S71.
\end{abstract}

Data sources Sources were Medline and the Cochrane Oral Health Group Specialist Register up to April 2001. Only English-language studies were included.

Study selection Controlled trials and longitudinal studies, with data analysed at patient level, were chosen for consideration.

Data extraction and synthesis Information about the quality and characteristics of each study was extracted independently by two reviewers. Kappa scores determined their agreement. Data were pooled when mean differences and standard errors were available using a fixedeffects model.

Results No randomised controlled trials were identified. Four out of 10 of the controlled studies found that subgingival debridement (SGD) is clinically effective. In the one study where SGD was found to not be effective, oral hygiene instruction was not provided. The weighted mean of attachment gain of SGD in pockets that were initially $\geqslant 5 \mathrm{~mm}$ was $0.64 \mathrm{~mm}$, compared with $0.37 \mathrm{~mm}$ for supragingival plaque control (SPC) only. The reduction of pocket depth was 0.59 and $1.18 \mathrm{~mm}$ for SPC and SGD, respectively.

Eighteen papers only provided information on the effect of treatment compared with baseline values, eight showed SGD to be beneficial with regard to clinical attachment level change, and the remaining 10 provided no such an analysis. The weighted mean of this effect was a $0.74-\mathrm{mm}$ gain of attachment as a result of treatment in pockets initially $\geqslant 4 \mathrm{~mm}$.

Conclusions When people have chronic periodontitis, SGD (in conjunction with SPC) is an effective treatment in reducing probing pocket depth and improving the clinical attachment level. Further, it is more effective than SPC alone.

\section{Commentary}

This systematic review substantiates the findings of two other recent systematic reviews on the same topic. ${ }^{1,2}$ Consistently, scaling and root planing resulted in a decrease in pocket depth and an increase in attachment. This clinical benefit is directly related to the depth of the pocket being treated. The key exception is initially shallow pockets which lose attachment following scaling. Thus, these results validate a long-held clinical view that scaling is clinically effective.

In light of these results, two other systematic reviews reported in this issue of Evidence-Based Dentistry are noteworthy. The systematic review comparing powered and manual scaling ${ }^{3}$ indicates that both are equally effective. The systematic review of adjunctive systemic antimicrobials $^{4}$ suggests benefit from their use. Together, these three reviews begin to provide a new view of initial nonsurgical periodontal treatment. That is, scaling with powered instruments followed by adjunctive systemic antimicrobials. This view is both traditional and progressive. The educational and clinical traditionalists might say that the clinical effectiveness of scaling with hand instruments withstood the test of time and systemic antibiotics raise other risks. So, why change? The educational and clinical progressive might say that these approaches show promise, and may improve outcomes while reducing clinical effort, time and costs. So, why not try them?

Both views are defensible and valid. Thus, if evidence-based healthcare is the integration of clinical evidence with patient values and clinical experience, one can certainly use the current set of results for the first bit. The second bit will depend on the patient. The third, however, is tricky. It will rest on a clinician's ability to determine whether, when, and how they might integrate these new sets of data into their practice.

\section{Practice point}

- Subgingival debridement in conjunction with plaque control is effective in reducing probing pocket depth and improving the clinical attachment level.

\section{Richard Niederman}

DSM-Forsyth Center for Evidence-Based Dentistry, The Forsyth Institute, and the Department of Health Policy and Health Services Research, Boston University Goldman School of Dental Medicine, Boston, Massachusetts, USA

1. Elley J, Gold L, Burls A, Gray M. Scale and Polish for Chronic Periodontal Disease. Birmingham: West Midlands Health Technology Assessment Group, Department of Public Health and Epidemiology, University of Birmingham; 2001.

2. Hung HC, Douglass CW. Meta-analysis of the effect of scaling and root planing, surgical treatment and antibiotic therapies on periodontal probing depth of attachment loss. J Clin Periodontol 2002; 29:975-986.

3. Tunkel J, Heinecke A, Flemmig TF. A systematic review of efficacy of machinedriven and manual subgingival debridement in the treatment of chronic periodontitis. J Clin Periodontol 2002; 29(suppl. 3):S72-S81.

4. Herrera D, Sanz M, Jepsen S, Needleman I, Rolda'n S. A systematic review on the effect of systemic antimicrobials as an adjunct to scaling and root planing in periodontitis patients. J Clin Periodontol 2002; 29(suppl. 3):S136-S159.

Evidence-Based Dentistry (2003) 4, 61.

doi:10.1038/sj.ebd.6400211 